$\dot{\mathrm{T}}$. Hida

Nagoya Math. J.

Vol. 38 (1970), 13-19

\title{
NOTE ON THE INFINITE DIMENSIONAL LAPLACIAN OPERATOR
}

\author{
TAKEYUKI HIDA
}

To Professor Katuzi Ono on the occasion of his 60th birthday.

\section{\$0. Introduction.}

The infinite dimensional Laplacian operator can be discussed in connection with the infinite dimensional rotation group ([1]). Our interest centers entirely on observing how each one-parameter subgroup of the infinite dimensional rotation group contributes to the determination of the Laplacian operator.

We shall start with the measure of white noise. Let $E$ be a nuclear space of $C^{\infty}$-functions which is dense in $L^{2}\left(R^{1}\right)$ and satisfies the relation

$$
E \subset L^{2}\left(R^{1}\right) \subset E^{*},
$$

where $E^{*}$ stands for the dual space of $E$. Given a (characteristic) functional $C(\xi)=\exp \left(-\frac{1}{2}\|\xi\|^{2}\right),\|\xi\|$ being the $L^{2}\left(R^{1}\right)$-norm of $\xi \in E$, we can form a probability measure $\mu$ on $E^{*}$ such that

$$
C(\xi)=\int_{E^{*}} \exp [i\langle x, \xi\rangle] d \mu(x),
$$

where $\langle x, \xi\rangle, x \in E^{*}, \xi \in E$, is the continuous bilinear form which links $E$ and $E^{*}$. We call $\mu$ the measure of white noise.

By the infinite dimensional rotation group, we mean the group $O(E)$ which consists of all the linear transformations $g$ on $E$ satisfying the following two conditions:

i) Each $g$ is an isomorphism of $E$,

ii) $C(g \xi)=C(\xi)$ for every $\xi \in E$.

For each one-parameter subgroup $\left\{g_{t}\right\}$ of $O(E)$ we are given a unitary group $\left\{U_{t}\right\}$ in the following manner:

$$
U_{t} \varphi(x)=\varphi\left(g_{t}^{*} x\right), \varphi \in L^{2}\left(E^{*}, \mu\right),
$$

Received March 31, 1969 
where $g_{t}^{*}$ is the conjugate of $g_{t}$. With $\left\{U_{t}\right\}$ we can associate a generator $X$ :

$$
\left.\frac{d}{d t} U_{t}\right|_{t=0}=X
$$

We shall be interested in an operator $\Delta$ acting on $L^{2}\left(E^{*}, \mu\right)$ which enjoys the following properties:

i) $\Delta$ is a quadratic form of the $X^{\prime} s$,

ii) commutes with each $X$,

iii) annihilates constants,

iv) negative definite.

(cf. [2, Chapt. X]). It will be shown that such an operator $\Delta$ exists and is determined uniquely up to constant factor. Indeed, our $\Delta$ coincides with the infinite dimensional Laplacian operator given by Umemura [1].

In $\$ 2$ we shall see that finite dimensional rotations play a dominant role in the determination of $\Delta$ giving attention to the property (5) ii). However, to determine $\Delta$ completely we shall need quite different requirements arising from (5) iii) and iv). In fact, we shall make use of the feature of the support of $\mu$ (\$3).

Our method may not be the shortest way to obtain the explicit form of $\Delta$, however the discussion in this note seems to be helpful to carry on the harmonic analysis on the Hilbert space $L^{2}\left(E^{*}, \mu\right)$.

\section{§1. Preliminaries.}

Let $\left\{\xi_{n}, n \geq 1\right\}$ be a complete orthonormal system (c. o. n. s.) in $L^{2}\left(R^{1}\right)$ such that each $\xi_{n}$ belongs to $E$, and let $\mu$ be the measure of white noise. A tame function based on $\left\{\xi_{n}\right\}$ is a function on $\left(E^{*}, \mu\right)$ expressed in the form $f\left(\left\langle x, \xi_{1}\right\rangle, \cdots,\left\langle x, \xi_{p}\right\rangle\right)$ by a function $f$ on $R^{p}$ for some $p>0$.

For a strongly continuous one-parameter subgroup $\left\{g_{t}, t\right.$ real $\}$ we define the generator $A$ :

$$
A=\left.\frac{d}{d t} g_{t}\right|_{t=0}
$$

The unitary group $\left\{U_{t}\right\}$ and its generator $X$ are given by (3) and (4). We now introduce the operator $\frac{\partial}{\partial \xi_{j}}$ : If $\varphi(x)=f\left(\left\langle x, \xi_{1}\right\rangle,\left\langle x, \xi_{2}\right\rangle, \cdots\right)$, then 
$\frac{\partial}{\partial \xi_{j}} \varphi$ is given by $\left(\frac{\partial}{\partial \xi_{1}} \varphi\right)(x)=\left.\frac{\partial}{\partial t_{j}} f\left(t_{1}, t_{2}, \cdots\right)\right|_{t_{j}=<x, \xi_{j}>}$. By a formal computation we have the following assertion.

Proposition 1. Suppose that $A \xi_{n} \in E$ for every $n$. Then, for a tame function $\varphi(x)$ based on $\left\{\xi_{n}\right\}$, the generator $X$ of the unitary group $\left\{U_{t}\right\}$ is expressed in the form

$$
(X \varphi)(x)=\sum_{j}\left\langle x, A \xi_{j}\right\rangle\left(\frac{\partial}{\partial \xi_{j}} \varphi\right)(x) .
$$

To avoid notational complication, we sometimes use the notations $\varphi_{j}, \varphi_{j k}$, . to ednote $\frac{\partial}{\partial \xi_{j}} \varphi, \frac{\partial^{2}}{\partial \xi_{j} \partial \xi_{k}} \varphi, \cdots$

We now come to a consideration of a quadratic form of the $X$ 's of the form (7). Let $X$ and $Y$ be generators of unitary groups corresponding to one-parameter groups $\left\{g_{t}\right\}$ and $\left\{h_{t}\right\}$ with generators $A$ and $B$, respectively. Suppose that $A \xi_{j} \in E$ and $B \xi_{j} \in E$ for every $j$. Set

$$
A \xi_{j}=\sum_{p} \lambda_{j p} \xi_{p} \text { and } B \xi_{k}=\sum_{q} \nu_{k q} \xi_{q} .
$$

Then we have a formal expression

$$
(X Y) \varphi(x)=\sum_{k j} \alpha^{j k}(x) \varphi_{j k}(x)+\sum_{j} \beta^{j}(x) \varphi_{j}(x)
$$

for a tame function $\varphi$, where

$$
\alpha^{j k}(x)=\sum_{p q} \lambda_{j p} \nu_{k q}\left\langle x, \xi_{p}\right\rangle\left\langle x, \xi_{q}\right\rangle
$$

and

$$
\beta^{j}(x)=\sum_{k q} \lambda_{j k} \nu_{k q}\left\langle x, \xi_{q}\right\rangle .
$$

Thus a quadratic form $\Delta$ of the $X$ 's may be thought of as an operator expressed formally in the form

$$
\Delta=\sum_{j k} a^{j k}(x) \frac{\partial^{2}}{\partial \xi_{j} \partial \xi_{k}}+\sum_{j} b^{j}(x) \frac{\partial}{\partial \xi_{j}} .
$$

Noting the expressions of $\alpha^{j k}$ and $\beta^{j}$ in (8), $a^{j k}$ and $b^{j}$ in (9) must be the limits of quadratic forms and linear forms of the $\left\langle x, \xi_{n}\right\rangle, n \geq 1$, respectively. Now our problem can be stated as follows:

Starting out with the expression (9), determine the coefficients $a^{j k}(x)$ and 
$b^{j}(x)$ so that $\Delta$ satisfies all the conditions i) $\sim$ iv) in (5).

It is quite reasonable to assume that

(10) all the $a^{j k}(x)$ and $b^{j}(x)$ belong to the domains of $\frac{\partial}{\partial \xi_{p}}$ and $\frac{\partial^{2}}{\partial \xi_{p} \partial \xi_{q}}$, $p, q \geq 1$,

and that

$$
a^{j k}(x)=a^{k j}(x), j, k \geq 1
$$

\section{§2. Commutativity with finite dimensional rotations.}

In this section we shall find a necessary condition which is imposed upon the coefficients of $\Delta$ given by (9) by the requirement that $\Delta$ be commutative with finite dimensional rotations.

If $g \in O(E)$ acts in such a way that $g \xi=\xi$ for every $\xi$ orthogonal to some finite dimensional subspace of $E$, then $g$ is called a finite dimensional orthogonal transformation. The collection of such $g$ 's forms a subgroup of $O(E)$. We can also define a finite dimensional rotation in a similar manner.

An arbitrary finite dimensional rotation $g$ can be expressed as the product of two dimensional rotations via the Euler angles. Thus, in order that $\Delta$ be commutative with finite dimensional orthogonal transformations $\Delta$ must commute with two dimensional rotations. To be somewhat more specific let us take a two dimensional subspace spanned by $\xi_{p}$ and $\xi_{q}$, and let $g_{t}$ be the rotation through the angle $t$ in the plane $\left\{\xi_{p}, \xi_{q}\right\}$. With this choice of $g_{t}$ we are given a unitary group $\left\{U_{t}\right\}$ and its generator $X_{p q}$ represented in the form

$$
X_{p q}=\left\langle x, \xi_{p}\right\rangle \frac{\partial}{\partial \xi_{q}}-\left\langle x, \xi_{q}\right\rangle \frac{\partial}{\partial \xi_{p}}
$$

As in $\$ 1$, let $\left\{\xi_{n}\right\}$ be a c.o.n. s. in $L^{2}\left(R^{1}\right)$ such that $\xi_{n} \in E$ for every $n$.

Proposition 2. Suppose that the operator $\Delta$ given by (9) commutes with $X_{p q}$ for every pair $(p, q)$. Then we have

$$
\begin{aligned}
& a^{j k}(x)=c\left\langle x, \xi_{j}\right\rangle\left\langle x, \xi_{k}\right\rangle+\delta_{j, k} d, \quad j, k=1,2, \cdots, \\
& b^{j}(x)=b\left\langle x, \xi_{j}\right\rangle, \quad j=1,2, \cdots,
\end{aligned}
$$

where $b, c$ and $d$ are constants. 
Proof. The proof of (14) is quite easy. In fact, with a particular choice of $\varphi: \varphi(x)=\left\langle x, \xi_{q}\right\rangle$, the equation

$$
X_{p q} \Delta \varphi=\Delta X_{p q} \varphi
$$

implies that

$$
b^{p}(x)=\left\langle x, \xi_{p}\right\rangle b_{q}^{q}(x)-\left\langle x, \xi_{q}\right\rangle b_{p}^{q}(x) .
$$

Noting that $b^{p}(x)$ belongs to the span of the $\left\langle x, \xi_{n}\right\rangle$ 's, we see that $b_{q}^{q}$ is a constant independent of $q$ and that $b_{p}^{q}=0$ for $p \neq q$. Thus (14) is proved.

We proceed to the proof of (13). By using (14), the equation (15) for general $\varphi$ can be expressed in the form

$$
\begin{aligned}
& 2\left(\sum_{k} a^{p k}(x) \varphi_{q k}(x)-\sum_{k} a^{q k}(x) \varphi_{p k}(x)\right) \\
& \quad=\sum_{j, k} a_{q}^{j k}(x)\left\langle x, \xi_{p}\right\rangle \varphi_{j k}(x)-\sum_{j, k} a_{p}^{j k}(x)\left\langle x, \xi_{q}\right\rangle \varphi_{j k}(x) .
\end{aligned}
$$

Set $\varphi(x)=\left\langle x, \xi_{p}\right\rangle\left\langle x, \xi_{q}\right\rangle$, then we have

$$
a^{p p}(x)-a^{q q}(x)=X_{p q} a^{p q}(x) .
$$

If both $j$ and $k$ are different from $p$ and $q$, then we have

$$
X_{p q} a^{j k}(x)=0
$$

and for $k \neq q$ we have

$$
X_{j q} a^{j k}(x)=-a^{q k}(x) .
$$

Since $a^{j k}(x)$ is quadratic in $\left\langle x, \xi_{n}\right\rangle$ 's, direct computations of the relation (18) for all possible pairs $(p, q)$ enable us to obtain the expression

$$
a^{j k}(x)=a^{j k}\left(\left\langle x, \xi_{j}\right\rangle^{2}+\left\langle x, \xi_{k}\right\rangle^{2}\right)+c^{j k}\left\langle x, \xi_{j}\right\rangle\left\langle x, \xi_{k}\right\rangle+d^{j k} .
$$

For $j \neq k$ the relation (19) requires that $a^{j k}=0$. We may set $a^{j j}=0$. Finally, the equation (17) leads us to obtain $d^{p p}=d^{q q}$ and $c^{p p}=c^{q q}=c^{p q}$. Further, using (19) again, we see that $d^{j k}=0$ for $j \neq k$. Thus the equation (13) is proved.

So far we have just used the relation (15) to obtain the following formal expression:

$$
\Delta=c \sum_{j, k}\left\langle x, \xi_{j}\right\rangle\left\langle x, \xi_{k}\right\rangle \frac{\partial^{2}}{\partial \xi_{j} \partial \xi_{k}}+d \sum_{j} \frac{\partial^{2}}{\partial \xi_{j}^{2}}+b \sum_{j}\left\langle x, \xi_{j}\right\rangle \frac{\partial}{\partial \xi_{j}} .
$$


§3. Conclusion.

By a c. o. n. s. $\left\{\xi_{n} ; n \geq 1\right\}$ in $L^{2}\left(R^{1}\right)$ we are given a sequence $\left\{\left\langle x, \xi_{n}\right\rangle\right.$; $n \geq 1\}$ of mutually independent standard Gaussian random variables. The strong law of large numbers shows that

$$
\lim _{N \rightarrow \infty} \frac{1}{N} \sum_{n=1}^{N}\left\langle x, \xi_{n}\right\rangle^{2}=1 \text { for almost all } x \in E^{*}
$$

and that

$$
\lim _{N \rightarrow \infty} \frac{1}{N} \sum_{n=1}^{N}\left\langle x, \xi_{n}\right\rangle^{4}=3 \text { for almost all } x \in E^{*} .
$$

Now we can use the property (5) iii) which must be satisfied by $\Delta$ given by $\left(9^{\prime}\right)$. From $(20)$ and (21) the relations $\Delta 1=0$ and $\Delta 3=0$ imply the following equations:

$$
c+d+b=0, \text { and } 3 c+d+b=0,
$$

that is, $c=0$ and $b=-d$.

The negative difiniteness (5) iv) requires that for $\varphi(x)=\left\langle x, \xi_{1}\right\rangle$

$$
\int(\Delta \varphi(x)) \varphi(x) d \mu(x)=b \int\left\langle x, \xi_{1}\right\rangle^{2} d \mu(x)=b \leq 0
$$

must hold. To avoid trivial operator, the constant $b$ should be strictly negative: $b<0$.

Summing up the above discussions, we have

Theorem. If the operator $\Delta$ of the form (9) satisfies the conditions (5) i) iv), then

$$
\Delta=d \sum_{j}\left(\frac{\partial^{2}}{\partial \xi_{j}^{2}}-\left\langle x, \xi_{j}\right\rangle \frac{\partial}{\partial \xi_{j}}\right)
$$

with a positive constant $d$.

The operator given by $\left(9^{\prime \prime}\right)$ is exactly the same as the infinite dimensional Laplacian operator given by Umemura in [1]. In fact, the $\Delta$ given by $\left(9^{\prime \prime}\right)$ acts on $L^{2}\left(E^{*}, \mu\right)$ and its domain is rich enough including all the so-called Fourier-Hermite polynomials. It is interesting to note that the properties (20) and (21), that is the feature of so to speak the support of $\mu$, contribute in final determination of the infinite dimensional Laplacian operator. 


\section{REFERENCES}

[1] Y. Umemura, On the infinite dimensional Laplacian operator. J. Math. Kyoto Univ. 4 (1965), $477-492$.

[2] S. Helgason, Differential geometry and symmetric spaces. Academic Press. 1962.

[ 3 ] P. Lévy, Problèmes concrets d'analyse fonctionelle. Gauthier-Villars. 1951.

Mathematical Institute

Nagoya University 
